\title{
L'administration de la Preuve Face aux Exigences du Principe Dispositif en droit Civil Congolais
}

\author{
Malunga N'landu Ruth ${ }^{1}$
}

\section{Résumé}

Dans une affaire civile, le principe dispositif circonscrit l'étendu et le contenu des faits conformément à la requête des parties dans le cadre de l'instance. Il a donc pour fonction de venir déterminer l'office des parties quant aux faits. Cette fonction est présentée en parallèle avec celle du juge dans sa mission de dire le droit. Les parties doivent amener les faits dans le cadre de l'instance suivant les limites de la requête et le juge à son tours ne peut pas statuer au-delà des faits qui sous-tendent la requête qui lui est soumise par les parties, ni dénaturer les faits de la cause dans le procès civil. Ainsi, la preuve qui peut être exigée ou retenue à l'instance n'est relative qu'aux faits de la cause que les parties ont apportée devant le juge. Ni le juge, moins encore une partie au litige, ne peut exiger d'autres preuves ultra causa par respect au principe dispositif d'application en procédure civile en $R D C$ qui veut que se soit les parties qui amènent les faits à l'instance mais pas le juge et les preuves ne peuvent être exigé que dans la limite de ces faits. C'est dans ce contexte que cette étude s'intéresse aux défis auxquels est confrontée l'administration de la preuve face aux exigences du principe dispositif dans un procès civil en $R D C$.

\begin{abstract}
In a civil case, the device principle circumscribes the scope and content of the facts in accordance with the parties' motion in the proceeding. It therefore has the function of coming to determine the office of the parties as to the facts. This function is presented in parallel with that of the judge in his mission to say the right. The parties must bring the facts of the case within the limits of the application and the judge in turn can not rule beyond the facts underlying the request submitted to it by the parties, nor denature the facts of the case in the civil trial. Thus, the evidence that may be required or withheld from the proceeding is only the facts of the case that the parties brought before the judge. Neither the judge, let alone a party to the litigation, can demand other ultra causa evidence out of respect for the rule of civil procedure application in the DRC that wants the parties who bring the facts to the proceedings but not the judge and the evidence can only be required within these facts. It is in this context that this study focuses on the challenges faced by the administration of evidence against the requirements of the operative principle in a civil trial in the DRC.
\end{abstract}

1 MALUNGA N'LANDU Ruth est licenciée en Droit de l'Université de Kinshasa depuis 2015. Email : ruthmalunga5@gmail.com. 


\section{INTRODUCTION}

Le processus probatoire se trouve au cœur de la procédure ${ }^{2}$. Au-delà du droit pénal, il est présent en tout procès; car en justice, il ne suffit pas d'affirmer une prétention; encore fautil que celle-ci soit prouvée par des éléments suffisants pour espérer la conviction du juge sur le fondement des moyens invoqués par l'une ou l'autre partie. La preuve devient donc dans son principe une nécessité absolue en droit car, une partie qui veut prouver une prétention n'est pas libre de le faire comme elle l'entend au sens large. La nécessité de donner aux affaires judiciaires une garantie complète sûre et d'assurer aux plaideurs une de parfaite équité au débat et l'incertitude de preuve ont amené le législateur à réglementer l'administration de la preuve et en préciser les modes. Le législateur congolais a déterminé les procédés qui sont admis à l'exclusion de tous autres moyens. Celui-ci est même aller plus loin, il les a hiérarchisés en précisant que tel procédé qu'il détermine devait toujours avoir la préférence sur l'autre ${ }^{3}$.

En droit civil, le principe dispositif est en lien direct avec l'idée selon laquelle le procès est la chose des parties. Ce principe est classiquement associé au caractère accusatoire de la procédure. Il est défini comme signifiant que le procès est la chose des parties. Il peut aussi être défini comme « le principe selon lequel le procès est à la disposition des parties ». Dans cette perspective, le principe dispositif serait une traduction technique et juridique de l'idée selon laquelle le procès civil est la chose des parties. Selon ce principe, le contenu de la matière litigieuse est à la disposition des parties. Il en résulte que les parties ont la maitrise de l'étendu des faits de la cause, et que le juge a celle du droit. C'est dans ce contexte qu'il est admis, à chacun pour ce qui le concerne, que Da mihi factum, tibi dabo jus. Les parties ont la maitrise du déclenchement de la procédure, de son étendue, de son déroulement et de son extinction. Ainsi, en ce qui concerne l'administration de la preuve, les parties ne devraient être tenues d'apporter des preuves qu'aux faits qu'ils ont apportés à l'instance. Mais dans la pratique, le juge civil s'avère assez actif à tel point qu'il va au-delà du principe dispositif en apportant aussi certains faits mais dont la responsabilité de la charge de la preuve retombe sur les parties en dépit des exigences du procès civil en droit congolais et des principes qui régissent cette matière.

La problématique de la présente recherche est ainsi tiré du prescrit de l'article $31 \mathrm{du}$ code de procédure civile congolaise qui fait de la preuve contraire un droit en justice. De ce principe, il se dégage que doivent être prouvés, les faits concluants et contestés ${ }^{4}$ par la partie

2 Jean PRADEL, « Rapport général », Actes du séminaire international organisé par l'Institut supérieur international de sciences criminelles à Syracuse (Italie) du 20 au 25 janvier 1992, sur la preuve en preuve procédure pénale comparée, in Revue internationale de droit pénal, Association Internationale de droit pénal, 63è année, nouvelle série, 1er et 2ème trimestres, France, 1992.

3 Chapitre VI du Titre I du DÉCRET du 30 juillet 1888 des contrats ou des obligations conventionnelles. (B.O., 1888, p. 109).

4 Sur ce point, voir l'analyse critique de l'exigence de contestation du Professeur Lagarde, Lagarde (X.), Réflexion critique sur le droit de la preuve, op. cit., p.140, n88 et suiv. 
adverse; car en droit civil avons-nous rappelé, les prérogatives du juge en matière de preuve ne lui permettent pas de s'immiscer dans le fait. Le juge ne peut pas non plus introduire de nouveaux faits dans le débat. Il ne dénature pas les prétentions des parties. Il n'instruit pas à vocation de découvrir de nouveaux faits; car, il n'est pas de son rôle de prouver les faits que les parties ont allégués ou non mais qui se trouvent dans le débat. Tel est l'exigence du principe dispositif.

C'est pourquoi cette étude, tout en analysant les différents modes d'administration de la preuve dans une instance civile en RDC (A), s'emploiera à dégager et à critiquer le rapport qui existe entre la preuve et le principe dispositif dans un procès civil en droit congolais en ce référent aux cas pratique à travers des décisions judiciaires (B).

\section{A. L'administration de la preuve dans une instance civile en droit congolais}

Une instance est une suite d'actes de procédure allant de la demande en justice jusqu'au jugement. A l'ouverture de l'instance, il appartient) chaque partie d'alléguer les faits juridiques qui justifient sa demande. Les faits allégués qui ne sont pas contestés par la partie adverse, ne sont plus à prouver, le juge peut généralement les considérer comme établi en fait comme en droit. L'absence de contestation peut constituer un aveu implicite à l'égard de ces faits. Mais, il faut tout de même noter que le juge ne retiendra cependant pas les faits qui sont contraires aux faits notoires ou aux faits d'expérience commune. Il n'acceptera pas non plus pour acquis des faits, même expressément reconnus, s'ils sont contradictoires en saine logique. C'est lorsque les faits sont contestés que la partie qui les allègue doit en rapporter la preuve (actor incumbit probatio) ${ }^{5}$.

Les faits de la cause peuvent se partager en fait matériels ayant une incidence sur le rapport de droit des parties et en actes juridiques, c'est-à-dire des actes ayant été posés en vue de produire des effets juridiques notamment les conventions entre parties. Ainsi, c'est aux faits matériels et aux faits juridiques contestés que les parties sont demandées à apporter la preuve. En droit congolais, il est actuellement admis cinq modes de preuve. Il s'agit de la preuve littérale, la preuve testimoniale, les présomptions, l'aveu de la partie et le serment $^{6}$. Tout en suivant les règles d'usage, les parties peuvent prouver les faits qu'ils allèguent en utilisant un de ces différents modes de preuve.

Dans une instance civile l'on peut donc déduire que le juge ne peut former sa conviction par les procédés de preuve prévus par la loi et même hiérarchisés, le juge ne pourra trancher le débat par des connaissances personnelles sur les faits. En outre le juge est passif sur les débats qui s'ouvrent entre le demandeur et le défendeur. Ce sont donc les parties qui sont les véritables acteurs du débat judiciaire et ceux sont-elles qui administrent des preuves

5 Jean Carbonnier, Droit civil, Introduction, $n^{\circ} 173$, p. 298.

6 Article 198 du Décret du 30 juillet 1888 portant sur les Contrats et obligations conventionnelles. 
dans la mesure où elles estiment devoir ou pouvoir le faire pour débattre. Le juge se contente d'entendre et d'apprécier les preuves et les critiques fournies par les parties ${ }^{7}$.

\section{La preuve littérale}

La preuve littérale est l'ensemble des documents écrits qui concourent à l'administration de la preuve lorsque les circonstances l'exigent. Le droit congolais distingue les actes authentiques et les actes sous seing privé.

\section{Les actes authentiques}

En droit congolais les actes authentiques sont dénomme «titre authentique ${ }^{8}$ ». Ce dernier est celui qui a été reçu par un officier public ayant le droit d'instrumenter dans le lieu où l'acte a été rédigé, et avec les solennités requises ${ }^{9}$. Il est établi par un officier public (notaire par ex.), dont les affirmations font foi jusqu'à inscription en faux et dont les grosses, revêtues de la formule exécutoire, sont susceptibles d'exécution forcée. L'acte fait foi jusqu'à preuve du contraire des faits qui n'ont pas été personnellement constatés par l'officier public. Lorsqu'il est établi par un notaire, l'acte authentique est dispensé de toute mention manuscrite exigée par la loi, sauf disposition contraire expresse ${ }^{10}$.

L'acte authentique fait foi de la convention qu'il renferme entre les parties contractantes et leurs héritiers ou ayants cause jusque preuve littérale contraire. En cas de l'incompétence ou de l'incapacité de l'officier, ou par défaut de forme, l'acte reste considéré comme écriture privée s'il a été signé par les parties.

L'acte authentique s'oppose aux actes dits " sous seing privé ", soit les contrats, accords et conventions que les parties rédigent seules ou avec l'assistance de leurs conseils (avocat, juriste, fiduciaire, etc.), mais sans l'intervention d'un notaire. Mais il faut noter que les actes notariés ne sont pas les seuls actes authentiques.

Ainsi défini, on a l'image du vaste champ que couvre l'acte authentique : actes politiques et administratifs émis par le pouvoir public soit législatif. L'acte authentique fait preuve de son contenu sans qu'il soit nécessaire d'en prouver la signature, non plus que le sceau qui y est attaché ni le caractère de l'officier public qui l'a attesté. Alors que l'on ne peut attaquer l'acte authentique que par la procédure de l'inscription en faux, il est possible d'attaquer l'écrit sous seing privé par n'importe quel moyen; et si l'écrit sous seing privé vient à disparaître, on peut éprouver de grandes difficultés à prouver ce qu'il était destiné à constater ${ }^{11}$.

7 Anne Marie Leroyer, Les fictions juridiques, Thèse Paris II, 1995, p. 186.

8 Décret du 30 juillet 1888 , note 3.

9 Article 199 du Décret du 30 juillet 1888 note 3.

10 Serge Guinchard et Thierry debard (dir.), Lexique des termes juridiues, Paris, 2017, p.59.

11 Anne Marie Leroyer, note 7, p.28. 


\section{L'acte sous seing privé}

L'acte sous-seing privé est généralement instrumentaire, plus rarement nécessaire à l'existence de la situation juridique, rédigé par un particulier et comportant la signature des parties. Il n'a de force probante qu'autant qu'il n'y a pas dénégation ou méconnaissance de l'écriture ou de la signature par celui auquel on l'oppose, à moins que la partie qui s'en prévaut n'en démontre la sincérité.

Contrairement aux actes authentiques, les actes sous seing privé ne sont pas soumis à beaucoup de règles de formalisme. Certains actes sont soumis à plus de conditions de forme que d'autres mais les parties doivent dans tous les cas apposer leur signature sur l'acte.

Lorsque les actes contiennent des conventions synallagmatiques, il est prévu qu'ils ne sont valables qu'autant qu'ils ont été faits en autant d'originaux qu'il y a de parties ayant un intérêt distinct. Il suffit d'un original pour toutes les personnes ayant le même intérêt. Chaque original doit contenir la mention du nombre des originaux qui en ont été faits ${ }^{12}$. Le billet ou la promesse sous seing privé par lequel une seule partie s'engage envers l'autre à lui payer une somme d'argent ou une chose appréciable, doit être écrit en entier de la main de celui qui le souscrit; ou du moins il faut qu'outre sa signature, il ait écrit de sa main un bon ou un approuvé, portant en toutes lettres la somme ou la quantité de la chose ${ }^{13}$.

L'acte sous seing privé, reconnu par celui auquel on l'oppose ou légalement tenu pour reconnu a, entre ceux qui l'ont souscrit et entre leurs héritiers et ayants cause, la même foi que l'acte authentique. Celui auquel on oppose un acte sous seing privé est obligé d'avouer ou de désavouer formellement son écriture ou sa signature. Ses héritiers ou ayants cause peuvent se contenter de déclarer qu'ils ne connaissent point l'écriture ou la signature de leur auteur. Dans le cas où la partie désavoue son écriture ou sa signature, et dans le cas où ses héritiers ou ayants cause déclarent ne les point connaître, la vérification en est ordonnée en justice ${ }^{14}$.

\section{La preuve testimoniale en droit congolais}

La preuve testimoniale fait intervenir un témoin dont le témoignage constitue un moyen de preuve des faits allégué. Le témoignage est un acte par lequel une personne atteste l'existence d'un fait dont elle a eu personnellement connaissance, et non indirectement par ouïdire.

Toute personne qui est victime ou a été témoin d'un fait peut témoigner. Mais le témoin doit être en mesure de rapporter des faits au tribunal, c'est-à-dire des choses qu'il a luimême constatées ou entendues. Il ne doit pas donner son opinion. Le témoin doit aussi être

12 Article 207 du Décret du 30 juillet 1888, note 3.

13 Charles Perelman et Pierre Foriers, Les présomptions et les fictions, Bruxelles, 1974, p. 340.

14 Article 206 du Décret du 30 juillet 1888, note 3. 
capable de répondre aux questions des avocats. Il vient attester solennellement la véracité d'un fait ou d'une situation.

Ainsi, le témoin est un particulier invité à déposer, dans le cadre d'une instance ou sous la forme écrite d'une attestation, sur les faits dont il a eu personnellement connaissance, après avoir prêté serment de dire la vérité. Les personnes frappées d'une incapacité de témoigner peuvent cependant être entendues mais sans prestation de serment. Les témoins doivent faire connaître, s'il y a lieu, leur lien de parenté ou d'alliance avec les parties, de subordination à leur égard, de collaboration ou de communauté d'intérêts avec elles ${ }^{15}$.

En droit congolais, la preuve testimoniale ne pas admise comme preuve des créances de sommes d'argent d'une certaine valeur ${ }^{16}$. Néanmoins, les engagements commerciaux pourront être constatés par la preuve testimoniale dans tous les cas où le tribunal croira devoir l'admettre.

\section{Les présomptions comme mode de preuve en droit civil congolais}

Autre moyen mis au service de la sécurité juridique, les présomptions sont définies comme une opération de l'esprit par laquelle on admet l'existence d'un fait qui n'est pas directement démontré mais qui est rendu vraisemblable par la preuve supposée ou rapportée d'un autre fait.

Cette définition présente les présomptions comme à la fois un mode de preuve et un moyen de prouver, ceux-ci correspondant à un mode de démonstration. Les présomptions sont des conséquences que la loi ou le magistrat tire d'un fait connu à un fait inconnu. La présomption suppose un déplacement du fait à prouver, l'objet de la preuve glissant du fait qu'il s'agit en définitive d'établir à un autre fait qu'il suffira de démontrer à sa place. Au lieu de prouver directement le fait $\mathrm{A}$, on se contente de la preuve du fait $\mathrm{B}$ qui rend $\mathrm{A}$ vraisemblable. Le raisonnement est à la fois inductif et déductif et peut transiter par un principe général. Les exemples ne manquent pas : «Quand l'herbe bouge c'est qu'il y a du vent » dit le proverbe chinois cité par tous les auteurs qui ont travaillé sur la question ${ }^{17}$. Chacun sait aussi, que de l'existence du mariage au moment de la conception on induit la règle générale selon laquelle le mariage suppose un devoir de cohabitation et de fidélité et que de cette règle l'on déduit que le mari est présumé le père des enfants à venir de cette union. La présomption est un remède à une difficulté de preuve car le plus souvent le fait présumé est plus difficile à prouver que le fait retenu.

Les présomptions n'ont pas toutes la même origine. Certaines sont établies par la loi ${ }^{18}$. Dans ce cas, elles “ ne fournissent pas des éléments, mais dispensent de la preuve celui à

15 Jean Carbonnier, note 5, p.78.

16 Article 217 du Décret du 30 juillet 1888, note 3.

17 Jean Carbonnier, note 5, p.107.

18 Article 226, 227 et 228 du Décret du 30 juillet 1888, note 3. 
qui elles profitent ${ }^{19}$. A titre d'exemple, la bonne foi est toujours présumée et c'est à celui qui allègue la mauvaise foi à la prouver. Ces présomptions constituent des dispenses de preuve dans la mesure où elles imposent au juge de tenir les faits pour établis faute d'une démonstration contraire suffisante ${ }^{20}$. En droit congolais, il est prévu aussi des présomption laissées à l'appréciation du magistrat ${ }^{21}$. On peut aussi classer les présomptions suivant qu'elles sont simples ou absolues. Les premières opèrent un déplacement de la charge de la preuve. La contre-preuve peut l'emporter et ruiner la présomption si elle est plus convaincante. On dit aussi que la présomption opère un renversement de la charge de la preuve : le risque de la non-preuve pèse sur celui dont la preuve contraire est attendue. Si la présomption n'est pas susceptible de preuve contraire on dira qu'elle est irréfragable, elle confine à la fiction. Ce n'est plus une règle descriptive suivant laquelle il est possible d'établir la preuve d'une situation juridique qui a pu effectivement se réaliser, c'est un acte de volonté qui affirme un fait, peu importe que celui-ci soit faux ou non. La vérité du fait n'est plus seulement présumée mais avérée. On retrouve aux côtés de ces dernières, des présomptions qui quant eux ses veulent réfrangibles,

\section{Les aveux des parties comme mode de preuve dans un procès civil}

L'aveu est une déclaration par laquelle l'une des parties reconnait l'exactitude d'un fait qui lui est défavorable et qui est allégué par son adversaire. Le principe c'est que l'homme n'est pas censé mentir contre ses intérêts. On distingue deux types d'aveu qui n'ont pas la même force probante: l'aveu judiciaire et l'aveu extra judiciaire ${ }^{22}$.

On dit que l'aveu est judiciaire lorsqu'il est fait en justice devant un juge. Dans ce cas, il est irrévocable et indivisible (doit être indivisible). Il a une force probante absolue, la loi prévoit que le juge est obligé de le tenir pour vrai en matière civil. Si A avoue être débiteur de B mais qu'il ajoute tout de suite qu'il a payé sa dette, B ne peut pas diviser l'aveu de A en ne retenant la première partie. L'aveu judiciaire a une force probante absolue, la loi prévoit que le juge est obligé de le tenir pour vrai ${ }^{23}$.

Comme son nom l'indique, l'aveu extrajudiciaire est celui qui est fait hors de la présence du juge. Il peut être contenu dans tout document écrit émanant d'une des parties et dont le juge viendrait à prendre connaissance. Par exemple: une lettre missive. L'aveu extra-judiciaire n'a pas la même force probante, il ne lie pas le juge qui comme pour le témoignage ou la présomption judiciaire est libre de déterminer le crédit qu'il convient d'octroyer à cet aveu ${ }^{24}$.

19 Charles Perelman et Pierre Foriers, Les présomptions et les fictions, Bruxelles, 1974, p. 340.

20 Chaim Perelman, Ethique et Droit, Bruxelles, 2009, p. 593.

21 Article 229 du décret du 30 juillet 1888 , note 3 .

22 Article 230 du décret du 30 juillet 1888 , note 3 .

23 Article 230 du décret du 30 juillet 1888 , note 3 .

24 Cossette Laurent, Des actes authentiques. Les Cahiers de droit, 1998, pp. 76-91. 


\section{La preuve par serment en droit civil}

Le serment est l'affirmation solennelle par une partie d'un fait qui lui est favorable. L'admission d'un tel mode de preuve peut surprendre car si longtemps on pouvait présumer qu'en jurant l'auteur du serment disait la vérité en raison du sentiment religieux. Aujourd'hui ce mode de preuve peut sembler naïf mais il reste tout de même que l'auteur d'un faux serment s'expose à des sanctions pénales. Le serment est le mode de preuve utilisé en ultime recours quand il n'existe plus aucun autre mode de preuve. Il existe deux types de serments, le serment décisoire et le serment supplétoire.

Le serment décisoire est celui déféré par l'un des plaideurs à son adversaire. En d'autres termes, il lui demande de jurer que ce qu'il dit est vrai. Trois attitudes sont alors possibles : soit il prête serment et il gagne son procès; soit il refuse de prêter serment et il perd son procès; ou soit il refuse de prêter serment mais demande à son adversaire de prêter serment de la réalité de sa prétention. La force probante du serment décisoire est absolue, le juge est lié, il doit donner raison à celui qui a prêté serment.

En droit congolais, il est aussi admis le serment déféré d'office. C'est le juge qui demande à une partie de prêter serment de la réalité de sa prétention lorsqu'il estime que les éléments de preuve rapportés par les parties ne sont pas suffisants pour remporter son intime conviction. On ne peut avoir ce recours qu'en complément d'enquête. Ce serment ne lie pas le juge.

\section{B. Rapports entre l'administration de la preuve et le principe dispositif dans une instance civile}

Il est important d'avoir à l'esprit le fait que dans une affaire civile, le principe dispositif confère aux parties le pouvoir d'ouvrir l'instance, en circonscrire les faits de la causes, voire même en décidé de la clôture du dossier. Aussi, au cours d'une instance civile, la preuve ne peut être exigée que pour les faits apportés devant le juge par les parties et dont la véracité est contestée par l'une des parties ou pour éclairer la religion du juge ${ }^{25}$. Ainsi, les parties disposant de l'instance, en détermine les faits de la cause et ce n'est qu'aux seuls faits qu'il peut qu'il peut y avoir lieu d'en exiger des preuves. Dans la pratique, il est constaté que dans une instance civile, le juge apporte des faits auxquels il exige des preuves de la part des parties. Cette réalité vient indubitablement à l'encontre du principe dispositif en matière civile. C'est ainsi qu'il sera utile pour ce second point d'examiner la manière dont les parties et leurs conseils se sont-ils comportés face à cette réalité.

Ce second point examine deux décisions de justice dont l'une, rendue par le tribunal de grande instance de Kinshasa Gombe sous RC 116. 051 (I); et l'autre, rendue par le Tribunal de Grande Instance de Kinshasa / Kinkole sous R.C 255 (II). 


\section{Décision rendue sous le RC 116.051}

A l'analyse de cette décision de justice du tribunal de grande instance de Kinshasa/Gombe, l'attention sera focalisée sur l'application du principe dispositif dans la présentation des preuves par les parties. Tout en présentant les faits de la cause (1), il sera question de faire une critique de l'application du principe dispositif dans l'administration des preuves (2).

1. Résumé des faits de la cause

Ce fut Madame NGALULA TSHIMPANGA Nathalie contre monsieur NKONDO NDAYE Jean Paul sous RC 116.051, jugée devant le Tribunal de Grande Instance de Kinshasa Gombe. La requérante Madame NGALULA TSHIMPANGA Nathalie fut légalement mariée au feu NDAYE NKONDO MULEKELAY Laurent depuis le 19 septembre 2008 sous le régime de la communauté universelle des biens et ce, conformément à l'acte de mariage produit par elle, n¹53/2008; Volume II/2008; Folio 153/2008;

Que de leur union, outre les enfants nés dans le mariage, le couple a acquis plusieurs biens meubles et immeubles ${ }^{26}$. la loi n87-010 du 1er août 1987 telle que modifiée et complétée par la loi $n^{\circ} 16 / 008$ du 15 juillet 2016 portant code de la famille de la République Démocratique du Congo dispose que le régime de la communauté universelle consacre entre les époux la communauté de tous les biens, tant meubles qu'immeubles ainsi que de leurs dettes présentes et à venir. Cette même loi renchérit, que nonobstant toute coutume contraire, le mariage se dissout de plein droit par la mort de l'un des époux. ${ }^{27}$ Ainsi en droit Congolais, à la dissolution du mariage, l'actif et le passif de la communauté sont partagés par moitié entre les anciens époux ou entre le conjoint survivant et les héritiers de l'autre époux. ${ }^{28}$

\section{1. Biens immeubles}

1. Une parcelle sise $n^{\circ} 10$, de l'avenue Riviera dans la commune de Ngaliema couverte par un certificat d'enregistrement $n^{\circ} 18971$, Vol. AI 468, Folio 21.

2. Une parcelle sise avenue KINKOLE $n^{\circ} 16$ dans la commune de KANANGA, ville de KANANGA couvert par un certificat d'enregistrement Vol. KI 66 Folio 024.

3. Une parcelle sise $n^{\circ} 29645$ couverte par un contrat de location $n^{\circ} \mathrm{T} / 18.128$ dans le lotissement dialogue inter congolais, commune de la N'sele à Kinshasa.

2. Biens meubles

1. une jeep NISSAN Patrol, plaque 8400 BK.

2. une voiture MITSHUBISHI, plaque $8900 \mathrm{AL}$.

3. un camion Mercedes Benz 1017, plaque 9477 AI.

4. deux groupes électrogènes de 54 et de 25 KVA.

5. les équipements de gymnastique.

6. deux salons.

7. un tapis roulant.

27 Art. 541 et 538 point 1 de la loi la loi n87-010 du ler août 1987 telle que modifiée et complétée par la loi $n^{\circ} 16 / 008$ du 15 juillet 2016 portant code de la famille de la République Démocratique du Congo.

28 Article 535 al 1 de la loi ci-dessus évoquée. 
Ayant brandi l'acte de décès nD170969733-02 attestant la mort de son mari monsieur NDAYE NKONDO MULEKELAY Laurent en date du 03/04/2017 au Canada, à Montréal, elle a sollicitée du tribunal de séant de faire application intégrale de toute les dispositions légales sus dessus évoquées; C'est-à-dire, de prononcer au préalable la liquidation du régime matrimonial de la communauté universelle des biens des époux, ainsi d'ordonner le partage des biens par moitié.

Ayant reçu l'offre d'une faramineuse somme de la partie défenderesse, dans sa décision rendue le 15/juin/2018, le tribunal de céans opta pour dénaturer les faits et ce, sansla moindre motivation ${ }^{29}$, ignora la preuve de l'acte de mariage produite en bonne et due forme par la requérante sans qu'au préalable, il ne soit attaqué en faux au motif qu'elle n'avait pas visité la composition après plaidoirie. Et pourtant, l'article 434 du code de la famille de la République Démocratique du Congo dispose que l'acte du mariage produit ses effets à l'égard de tous. Donc cet acte lie le juge jusqu'en l'inscription en faux. Ainsi, « il appartient à celui qui allègue leur fausseté de démontrer que ce mariage n’a pas été célébré ou enregistré, soit qu'il a été célébré ou enregistré à une date autre que celle résultant de ces actes ». Dans le même sens, l'article 435 du même code renchérit « qu'il appartient à celui qui allègue qu'un mariage a été déclaré nul ou a été dissout d'en apporter la preuve ».

Ayant été notifiée de la décision désagréable hors du droit, la requérante par la voie de ses conseils releva appel sous RCA 8759.Après examen minutieux des faits tels que restitués par la demanderesse, dans sa décision rendue en date du 02 Octobre 2018, la Cour d'Appel de Kinshasa/Gombe annule la décision rendue sous RC 116.051 et motive à la même occasion sa décision en des termes que «....les faits de la présente cause doivent être

29 En violation de l'art 21 al 1 de la Constitution du 18 février 2006 telle que modifiée par la loi no $11 / 002$ du 20 janvier 2011 qui dispose que " Tout jugement est...motivé », la plupart des jugements n'indiquent pas la base de leur décision ou autrement-dit le motif ou la motivation c'est-àdire les moyens de preuve par lesquels ils se sont convaincus (voir arts 353 et 485 al 1 et 2 du code de procédure pénale français). A titre exemplatif d'un jugement motivé en droit pénal congolais, il a été jugé que « Faute d'apporter la preuve que le prévenu, Commissaire de Police, avait été révoqué et n'était plus habilité à détenir en cette qualité une arme, l'infraction de port illégal d'arme à feu n'est pas établie à suffisance de preuve » C.A. Kin. R.P.A. 8783 du 4 février 1974, In RJZ 1979, No 1,2,3, p.103. Pour une idée éloquente de l'acuité avec laquelle le problème de motivation se pose en droit congolais, lire, notamment, DIBUNDA, K, M, Répertoire général de jurisprudence de la Cour Suprême de Justice 1969-1985, Ed. Connaissance et Pratique du Droit Zaïrois «C.P.D.Z. », Kinshasa, 1990, pp. 124-134. En effet, aux termes de l'art 21 al 1 de la Constitution congolaise du 18 février 2006 telle que modifiée par la Loi no 11/002 du 20 janvier 2011 portant révision de certains articles de la Constitution de la République Démocratique du Congo du 18 février 2006, tout jugement doit être motivé. Aux termes de l'art 485 du code de procédure pénale français, «Les motifs constituent la base de la décision ». A ce sujet, il a été jugé par le Conseil Constitutionnel français, que «l'obligation de motiver les jugements et arrêts de condamnation constitue une garantie légale du principe constitutionnel selon lequel la procédure pénale doit garantir contre toute forme d'arbitraire dans le jugement des affaires pénales ». Voir Conseil Constitutionnel, Communiqué de Presse 2011-113/115 QPC disponible à http://www.conseil-constitution nel.fr/conseilconstitutionnel/francais/les-decisions/acces-par-date/decisions-depuis-1959/2011/201 1-113/115qpc/communique-de-presse.95622.html consulté le 19 avril 2012. 
restitués de la manière suivante...». plus loin encore dans cette décision, la Cour d'appel renchérit «...........qu'il y a lieu de déclarer cet acte de mariage valable aussi longtemps qu'il n'a pas été attaqué en faux et de confirmer par cette preuve d'acte de mariage qu'il ne fait l'ombre d'aucun doute que madame NGALULA TSHIMPANGA Nathalie fut légalement mariée au feu NDAYE NKONDO MULEKELAY Laurent et par conséquent, mérite le bénéfice de l'application intégrale de l'article 535 al 1 du code de la famille qui consacre le partage par moitié des biens laissés par le De Cujus, son cher époux et ce, avant tout partage entre héritiers. $\gg{ }^{30}$.

\section{Critique sur la première décision}

La décision de la cour d'appel de Kinshasa Gombe a le mérite d'être louée car non seulement qu'elle casse un mal jugé d'une juridiction inferieure, mais aussi et surtout corrige le comportement inapproprié des certains juges qui se soldent face à l'argent, pour dénaturer les faits du litige leur soumis, c'est-à-dire de violer le principe dispositif, quand bien même que la victime aura produit une preuve redoutable, en l'espèce l'acte de mariage.

\section{De la deuxième décision rendue sous R.P. 255}

1. résumé des faits de la cause

Dans cette affaire qui opposa monsieur BAMUABI BALEJA Matony contre ITONGO DJEMELAS Jean-Claude devant le tribunal de Grande Instance de Kinshasa/Kinkole sous RP. 255, le fait se présenta comme suit :

Madame DJEMELASNYAMAPATI mamie est propriétaire d'une parcelle de terre située au numéro 23, de l'avenue Djemelas, quartier Badara, commune de la N'sele; de ce fait, voisin de monsieur BAMUABI BALEJA a qui il reproche d'avoir occupé illégalement le deux mettre de sa parcelle ainsi que le quatre autre mettre sur l'espace publique. Déposa à l'appui de ses accusations, l'assignation au nom de son petit frère ITONGO DJEMELAS Jean-Claude munie des pièces soutenant son accusation, au greffe du tribunal de céans. Devant ce Tribunal, le cité monsieur BAMUABI BALEJA souleva plusieurs exceptions notamment :

a) Nul ne plaide par procureur

Selon le cité BAMWABI BALEJA MATONNY, ce principe général de droit veut en ce qu'aucune personne ne parle au nom d'un tiers sans le mandat de ce dernier; qu'en l'es-

30 Article 535 de la loi nº $87-010$ du 1er août 1987 telle que modifiée et complétée par la loi n $16 / 008$ du 15 juillet 2016 portant code de la famille de la République Démocratique du Congo dispose « qu'à la dissolution du mariage, l'actif et le passif de la communauté sont partagés par moitié entre les anciens époux ou entre le conjoint survivant et les héritiers de l'autre époux ». 
pèce, il pria au Tribunal de retenir que le fait pour le citant de revendiquer ce qu'il appelle emprise de l'état sans qu'il ne produise la procuration spéciale de l'état congolais l'autorisant d'agir à son nom et pour son compte en justice contre le cité ouvre la voie au tribunal de céans de rejeter cette action; car, dépourvu de droit de propriété de l'espace prétendument occupé et donc non préjudicié par l'occupation du cité. Car, à la lumière de l'article 54 du Code de Procédure Pénal de la République démocratique du Congo, il ressort que la requête en l'espèce ne peut être faite qu'à l'initiative de la partie lésée qui est l'Etat congolais, et non du citant DJEMELAS.

A en croire le BAMUABI, l'emprise publique fait partie intégrante du domaine publique de l'Etat, qu'en tant que tel, son occupation illégale soit elle ne peut être revendiquée que par l'Etat seul, par le truchement du ministre de la justice ou du président de la République. D'autant pour dire que le fait pour DJEMELAS le citant de réclamer l'emprise dont il prétend être occupé anarchiquement par le cité BAMWABI, il se met sans mandat en lieu et place de l'Etat Congolais, seul propriétaire de l'emprise. Ce qui n'est pas permit.

En second aspect du défaut de qualité, le cité BAMUABI souleva in limine litis un moyen d'ordre publique lié à la qualité de monsieur ITONGO DJEMELAS Jean-Claude, petit frère du citant, qui selon le cité prétend représenter sa sœur en justice sans la moindre procuration spéciale. Et appuya ses allégations par une jurisprudence de la Cour Suprême de Justice ayant décidé que « une personne qui comparaitrait pour un être aux droits de cette personne et sans être mandatée pour la représenter se verrait également opposer une fin de non-recevoir faute de qualité, en vertu de l'adage « nul ne plaide par procureur. Cette fin de non-recevoir peut être postulée par toute partie intéressée, elle doit être soulevée par le ministère publique et retenue d'office par le tribunal et ce, en tout état de cause $»^{31}$.

\section{b) De l'illicéité de la demande}

Outre le défaut de qualité, le cité BAMWABI souleva un autre moyen lié à l'illicéité de la demande; et eut définie la comme tout demande contraire à la loi ou aux bonnes mœurs. Que pour cela selon lui, toute personne qu'initie une action en justice doit au préalable se rendre a l'évidence que sa demande est conforme à la loi et aux bonne mœurs sous peine d'irrecevabilité. Selon BAMWABI, la prétendue infraction d'occupation anarchique de l'emprise publique n'existant en aucune loi de la république démocratique du Congo, le Tribunal ne saura accorder d'égard aux allégations formulées « contra legem » et ce, en vertu de l'article 17 de la constitution de la $\mathrm{RDC}^{32}$.

31 Léo., 23 Aout 1932(R.J. 1933, p.91) repris dans l'ouvrage A.RUBBENS, Intitulé du droit judiciaire congolais,

Tome 2, presse universitaire du Congo, Kinshasa p. 87..

32 Constitution de la République Démocratique du Congo, modifiée par la Loi n 11/002 du 20 janvier 2011 portant révision de certains articles de la Constitution de la République Démocratique du Congo du 18 février 2006 (Textes coordonnés), Journal Officiel de la République Démocratique du Congo, numéro Spécial, Kinshasa, 5 février 2011. 
Dans le même sens, l'article 17 de la constitution de la République démocratique du Congo du 18 février 20006 telle que modifiée à ce jour dispose ce qui suit «..... Nul ne peut être poursuivi arrête, détenu, au condamne qu'en vertu de la loi et dans les formes qu'elle prescrit. Nul ne peut être poursuivi pour une action ou elle est commise et au moment des poursuites. Nul ne peut être condamne pour une action ou une omission qui ne constitue pas une infraction à la foi au moment où elle est commise et au moment de la condamnation .....»;

De l'analyse de cette disposition constitutionnelle en combinaison avec l'article premier du code pénal congolais ${ }^{33}$ qui prévoit le principe de la légalité des délits et peines, le cité à la même occasion prit eu tribunal de rendre a l'évidence que l'action mue par le citant est illicite au motif qu'à ce jour, l'occupation anarchique de l'emprise publique ne constitue pas une infraction en droit congolais.

Ayant reçue l'offre d'une faramineuse sommes là encore de la part du citant ITONGO DJEMELAS Jean-Claude qui n'a réussi à aucune phase du débat à produire une procuration spéciale lui remis par sa sœur Mamie DJEMELAS NYAMAPATY, preuve du fondement de son action en justice, dans sa décision du 05/07/2017, le Tribunal de Grande Instance de Kinshasa/Kinkole rendit a la surprise de tous, une décision mortelle en joignant tous les exceptions, voire même celle d'inconstitutionnalité au fond et renvoya la dite cause en prosecution pour plaidoirie deux semaine plus tard.

\section{Critique sur la deuxième décision}

A la suite de la prise de l'affaire en délibérer fut prononcée une année et trois mois plus tard le juge de la cause. Malgré le certificat d'enregistrement brandi par le cité BAMWABI, preuve de son installation de l'espace occupé, le reconnaissant entant que seul propriétaire de dudit espace face à la fíche parcellaire brandi par le citant ITONGO DJEMELAS, le Tribunal de Grande Instance de Kinshasa/Kinkole à ordonné l'annulation du certificat d'enregistrement détenu par le cité BAMWABI. Cela est allé au-delà de la demande telle que formuler par le citant Djemelas qui reclamé seulement deux mettre occupé illégalement par l'autre partie. Dans cette état des choses, le juge n'a pas respecté les exigences du principe dispositif tel que consacré.

Ayant été notifiée de la décision qui annule son certificat d'enregistrement, monsieur BAMWABI interjeta appel dans le délai devant la Cour d'appel de Kinshasa/Matete sous RCA 1596, laquelle cause dont après examen et descente sur le lieu, la Cour remettra le cité dans ces droits et ordonna que le deux mettre de l'espace réclame par monsieur ITONGO le citant, soit divisé à moitié et que chacune de parties occupe un mettre de longueur. Décision qui a

33 Décret du 30 janvier 1940 tel que modifié et complété par la loi de 2004 portant modification du Code pénal Congolais. 


\section{CONCLUSION}

La présente étude a porté sur l'administration de la preuve dans une instance civile face aux exigences du principe dispositif d'application en droit civil congolais. La compréhension de cette étude à été soutenue par l'analyse de deux jugement à l'appui des considérations théoriques. La première décision a été rendue par le Tribunal de grande instance de Kinshasa/ Gombe et la seconde, par le Tribunal de grande instance de Kinshasa / Kinkole pour comprendre la manière dont le juge congolais perçoit devant lui la notion de l'administration de la preuve telle que prévue en matière civile face aux exigences du principe dispositif.

Prenant en compte des considérations théoriques, il ressort de la première décision que le juge de grande instance congolais fournit d'effort suffisante pour le respect du principe dispositif en laissant au parties en cause de donner les faits et d'en apporter les preuves si nécessaire. Par contre, la seconde décision quant à elle semblait ne pas prendre en compte les limites du principe dispositif dans l'administration de preuves pour soutenir les prétentions des parties. La grande difficulté reste la vigilance et les connaissances des parties et leurs avocats pour demander au juge de ne pas s'immiscer dans les prérogatives des parties en ce qui concerne le contenu et la limitation de la demande, mais aussi dans la production des éléments de preuve pour soutenir les demandes de chaque partie.

\section{BIBLIOGRAPHIE}

\section{Textes juridiques}

Arrêté d'organisation judiciaire n² 299/79 portant règlement intérieur des cours, tribunaux et parquets, (Ministère de la Justice), Journal Officiel-Leganet.cd, disponible sur http://www.leganet.cd/Legisla tion/Droit\%20Judiciaire/AO.20.8.1979.htm consulté le 11 octobre 2012.

Charte africaine des droits de l'homme et des peuples, adoptée le 27 juin 1981 à Nairobi, au Kenya, lors de la 18 è Conférence de l'Organisation de l'Unité Africaine, entrée en vigueur le 21 octobre 1986, ratifiée par la RDC le 20 juillet 1987, Ordonnance-loi n 87-027 du 20 juillet 1987, Journal officiel, numéro spécial, septembre 1987, voir aussi Journal officiel, numéro spécial, 5 décembre 2002.

Constitution de la République Démocratique du Congo, modifiée par la Loi n 11/002 du 20 janvier 2011 portant révision de certains articles de la Constitution de la République Démocratique du Congo du 18 février 2006 (Textes coordonnés), Journal Officiel de la République Démocratique du Congo, numéro Spécial, Kinshasa, 5 février 2011.

Décret du 30 janvier 1940 portant Code pénal tel que modifié jusqu'au 31 décembre 2009 et ses dispositions complémentaires, Ministère de la Justice, RDC, 2010.

Décret du 30 juillet 1888 - Des contrats ou des obligations conventionnelles (B.O., 1888.

Loi la loi n87-010 du 1er août 1987 telle que modifiée et complétée par la loi n¹6/008 du 15 juillet 2016 portant code de la famille de la République Démocratique du Congo.

Ordonnance-loi 82-017 du 31 mars 1982 relative à la procédure devant la Cour suprême de justice, JOZ, 1er avril 1982, nº 7 . 


\section{Jurisprudence}

R.C 116.051 du 23 avril 2018, NGALULA TSHIMPANGA Nathalie C. NKONDO NDAYE Jean-Paul RP. 255 du 03 juin 2018, ITONGO DJEMELAS Jean-Claude C. BAMWABI BALEJA MATONY

C.A. Kin. R.P.A. 8783 du 4 février 1974, In RJZ 1979,

\section{Doctrine}

\section{A. Ouvrages}

BLANCHARD BONARD, les règles de l'Art à matière Civile, Paris, 2004.

BUCHAIB PINARD, Droit pénal Marocain, Casablanca, 2007.

YOKA MAMPUNGA Jean-Jacques., Codes congolais de procédure pénale, Kinshasa, 1996.

BOUZAT P., «La loyauté dans la recherche des preuves », Mélanges Legros, Sirey, 1964.

CHAMBON et GUERY, Droit et pratique de l'instruction préparatoire, Paris, 2007.

DIBUNDA KABUINJI MPUMB UAMBUJ, Répertoire général de jurisprudence de la Cour Suprême de Justice 1969-1985, Kinshasa, 1990.

IYELEZA MASIKA INSENGINGO, Recueil des textes constitutionnels de la République du Zaïre, du

19 mai 1960 ou 28 avril 1991 avec en annexe la charte coloniale du 18 oct. 1908, Kinshasa, 1981.

KAMIDI OFIT, Le système judiciaire congolais : organisation et compétence, Kinshasa, 1999.

\section{B. Articles}

CEDH, 23 juin 1993, Ruiz Mateos c/ Espagne, série A, n²62, §63; CEDH, 24 nov. 1997, Werner c/ Autriche, série $\mathrm{A}, \mathrm{n}^{\circ} 282$.

DIBUNDA KABUINJI MPUMB UAMBUJ, Répertoire général de jurisprudence de la Cour Suprême de Justice 1969-1985, Kinshasa, 1990.

GUINCHARD Serge (dir.), Droit processuel, Droits fondamentaux du procès, Paris, 1080.

Jean -Jaques DAIGRE, « La doctrine et la réforme de la procédure civile », JCPG, 1981.

KATUALA KABA KASHALA \& YENGI OLUNGU, Cour Suprême de justice: historique et textes annotés de procédure, Kinshasa, 2000.

KATUALA KABA KASHALA, Jurisprudences des Cours et Tribunaux 1975 - 1987, Kinshasa, Janvier 1992.

KATUALA KABA KASHALA, « Jurisprudence des cours et tribunaux, (19651974) », Kinshasa, RDC, Janvier 1992.

KATUALA KABA KASHALA, La preuve en droit congolais (textes, jurisprudence et doctrine), Kinshasa, 1998.

KATUALA KABA KASHALA, L'action publique, à travers, les jurisprudences et doctrine congolaise, belge et française, Kinshasa, 2004.

KENNES, $L$, Manuel de la preuve en matière pénale, Belgique, 2009.

MATADI NENGA GAMANDA, Le droit à un procès équitable, Kinshasa, 2002. 
PRADEL Jean, « Rapport général », Actes du séminaire international organisé par l’Institut supérieur international de sciences criminelles à Syracuse (Italie) du 20 au 25 janvier 1992, sur la preuve en preuve procédure pénale comparée, in Revue internationale de droit pénal, Association Internationale de droit pénal, 63è année, nouvelle série, 1er et 2ème trimestres, Ed Erès, France, 1992.

SOHIER Antoine, «Pratique des juridictions indigènes », notes sur l'application du décret du 15 avril 1926, Bruxelles, 1932. 